\title{
Metodología para la inclusión del uso de TICs en el aula en estudios relacionados con sostenibilidad
}

\section{Rosa Arroyo $^{a}$, Erika De Oliveira Jardim ${ }^{\mathrm{b}}$ y Vanesa G. Lo-Iacono-Ferreira ${ }^{\mathrm{c}}$}

${ }^{a}$ Departamento de Ingeniería e Infraestructura de los Transportes, Universitat Politècnica de València, maarlo3@cam.upv.es. ${ }^{b}$ Departamento de Química Inorgánica, Universidad de Alicante, erika.jardim@ua.es y ${ }^{c}$ Escuela Politécnica Superior de Alcoy Universitat Politècnica de València. valoia@upv.es

\begin{abstract}
This paper presents the methodology developed to include the use of ICTs as a tool for the acquisition of skills and competences related to sustainability in university courses. The main objective is to attract the interest and enthusiasm of the students in Sustainable Development, both in personal related aspects and during the development of their professional career. For this purpose, the use of ICTs tools is proposed, which will serve as a mean of communication between the students and the professor through several platforms and online applications, such as Google forms ${ }^{\circledR}$, Kahoot and those specific tools of each university. The methodology is composed of a guide for its implementation, an online survey, a game to carry out in class and an ad-hoc software for gathering additional specific information.
\end{abstract}

Keywords: methodololy, ICTs, gamification, Kahoot, Google Forms, sustainabiliy.

\section{Resumen}

Este trabajo presenta la metodología desarrollada para la inclusión del uso de las TICs como instrumento de apoyo para la adquisición de habilidades y competencias en el ámbito de los estudios de la sostenibilidad en el aula, en estudios universitarios. De esta manera, se pretende fomentar el interés y el entusiasmo de los estudiantes en el Desarrollo Sostenible, tanto en los aspectos más generales que afectan a la vida cotidiana como en el desempeño de la carrera profesional. Para ello, se propone el empleo de las TICs, como medio de comunicación entre el alumnado y el profesorado, a través de plataformas virtuales y herramientas on-line, como Google forms ${ }^{\circledR}$ y Kahoot ${ }^{\circledR}$, asi como otras propias de las propias universidades. La metodología presentada se compone de: una guía para su aplicación, una encuesta, un juego a desarrollar en el aula, y una herramienta propia para la recogida de datos.

Palabras clave: metodología, TICs, gamificación, Kahoot, Google Forms, sostenibilidad.

\section{Introducción}

La emergencia mundial, abarca no solo la salud de la población sino también la existencia del propio planeta tal como se conoce actualmente. Es de gran importancia que la sociedad reoriente su modelo productivo, su pensamiento y su propio sentido de la existencia para conservar el mundo tal como lo conocemos. La naturaleza ha de tener el papel central que merece orientando los procesos productivos a favor del medio ambiente y el desarrollo sostenible (Reyes-Sanchéz, 2012). 
La Educación para el Desarrollo Sostenible es la clave para formar a los futuros profesionales con una base sólida en responsabilidad ética y medio ambiente (Jardim, 2016 \& Annan-Diab, 2017). La importancia de la sostenibilidad ha de ser un elemento transversale en el aula. Desde la innovación educativa, el uso de las nuevas tecnologías de la información y las comunicaciones (TCIs) para fomentar nuevos escenarios de enseñanza-aprendizaje adquieren importancia (Ojeda-Barceló 2011, Hernández-Crespo et al., 2019).

La incorporación de los elementos lúdicos como parte el aprendizaje, permiten el aprendizaje de conceptos formales en un entorno incentivador favoreciendo los proceso cognitivos (Álvarez Romero \& Osete Cortina, 2019, Guardia et al., 2019). El alumnado evalúa positivamente la gamificación como elemento dinamizador en el aula (Ruiz et al., 2019; Pastor Villa, 2019). Las encuestas en línea (Google Forms $\left.{ }^{\circledR}\right)$ y los juegos de preguntas y respuestas (Kahoot $\left.{ }^{\circledR}\right)$ entre otros, han sido identificados como aliados en estas estrategias educativas (García-García et al., 2017; Chaiyo \& Nokham, 2017; Sainz-deAbajo et al., 2019).

Este trabajo evalúa el efecto de la inclusión del uso de las TICs como medio de instrucción en desarrollo de habilidades y competencias en el ámbito de los estudios de la sostenibilidad en el aula. Se propone un proyecto integrado de aplicación conformado por diversas herramientas TIC y de gamificación para favorecer la interiroización del Desarrollo Sostenible como concepto clave y transversal en toda carrera profesional. Con el fin de testear y validar este trabajo, durante el curso 2019/2020 se encuentra en proceso de aplicación en cuatro asignaturas (tres de ellas en la UPV y otra en la UA), con un número aproximado de 50 estudiantes participantes.

El presente trabajo se enmarca dentro del proyecto titulado "La inclusión del uso de las TICs como medio de instrucción en el desarrollo de habilidades y competencias en el ámbito de los estudios de la sostenibilidad en el aula" del Instituto de Ciencias de la Educación de la Univerisdad de Alicante (curso 2019/2020 - código 4947) en el que participa profesorado de la Universidad de Alicante (UA) y la Universitat Politècnica de València (UPV). Se presenta el marco del proyecto, definiendo en el objetivo las competencias transversales que abarcan las distintas asignaturas involucradas. Se describe el desarrollo del proyecto; cómo se ideó y se aborda el resultado del mismo haciendo hincapié en la estrategia para incorporar las distintas metodologías que forman parte de la iniciativa.

\section{Objetivos}

El objetivo de este trabajo es presentar la metodología desarrollada para la inclusión del uso de las TICs como instrumento de apoyo para la adquisición de habilidades y competencias en el ámbito de los estudios de la sostenibilidad en el aula.

A través de la aplicación de esta metodología, se pretende fomentar el interés y el entusiasmo de los estudiantes en el Desarrollo Sostenible, tanto en los aspectos más generales que afectan a la vida cotidiana como en el desempeño de la carrera profesional. El objetivo es que el alumnado comprenda la importancia de la sostenibilidad en la sociedad actual y futura con independencia de la titulación que cursa. Este enfoque, además, permite la adquisición de competencias transversales definidas de forma general por la institución donde se imparten las asignaturas. Estas competencias son:

- análisis y resolución de problemas (CT3, UPV y CT17, UA),

- responsabilidad ética, medioambiental y profesional (CT7, UPV y CT28, UA),

- pensamiento crítico (CT9, UPV, CT12, UA),

- conocimiento de problemas contemporáneos (CT10, UPV),

- competencias informáticas e informacionales (CGUA4). 
Esta metodología se encuentra en proceso de aplicación durante el curso 2019/2020 en cuatro asignaturas (tres de ellas en la UPV y otra en la UA). Las asignaturas han sido seleccionadas atendiendo a su relación con la sostenibilidad.

\section{Desarrollo de la innovación}

La innovación presentada en esta ponencia se basa en la inclusión de diferentes herramientas TICs como instrumento de apoyo para el trabajo de habilidades y competencias en el ámbito de los estudios de la sostenibilidad en el aula como parte de las competencias transversales.

Se propone el empleo de las TICs, como medio de comunicación entre el alumnado y el profesorado, a través de plataformas virtuales y herramientas on-line, como Google forms ${ }^{\circledR}$ y Kahoot ${ }^{\circledR}$, así como otras propias de las propias universidades. Los elementos TIC, por si solos, no aseguran la interiorización de contenidos y la dinamización de la clase al grado necesario para profundizar en conceptos que, sin ser ajenos completamente a las asignaturas, son más propios de las competencias transversales. Se plantea el uso conjunto de estas herramientas simultáneamente, de forma coordinada, con la realización de actividades tanto individuales como en grupo. Una guía detallada, planifica el uso de estas herramientas y articula las actividades que la relacionan. Por último, se propone también el desarrollo de un debate, desde distintos puntos de vista a respecto de lo que se puede aportar al un Desarrollo Sostenible:

- a título profesional (relacionado con formación, orientación de nuestro trabajo, en la toma de decisiones, etc.),

- a título personal (reciclaje, movilidad).

La metodología planteada consta de:

1. Una guía donde se describen las 8 fases que componen el proyecto para su aplicación docente.

2. Una encuesta en línea para evaluación de los conocimientos y percepción del alumnados al principio $\mathrm{y}$ al final del proyecto.

3. Un juego en línea para la interiorización de los conocimientos desarrollados.

4. Una herramienta de recopilación de información en línea para la evaluación y el debate de aspectos vinculaods a la movilidad de los alumnos.

El proyecto se desarrolla en dos sesiones de clase con duración aproximada de 2 horas cada una. Estas sesiones se llevan a cabo en dos etapas diferentes. La primera, dentro de las primeras sesiones de la asignatura $\left(5^{\mathrm{a}}\right.$ a $\left.7^{\mathrm{a}}\right)$, la segunda en las últimas sesiones de la asignatura $\left(25^{\mathrm{a}}\right.$ a $\left.28^{\mathrm{a}}\right)$; considerando una asignatura cuatrimestral desarrollada en 30 sesisones.

A continuación se describe en detalle cada uno de los elementos que componen la metodología como resultado de su diseño.

\section{Resultados}

Los elementos desarrollados para este proyecto cuentan con un elemento transversal integrador, la guía de aplicación. Esta guía detalla, en 8 etapas, la implementación completa del proyecto definiendo las actividades para cada una de las sesiones.

Dado el carácter de especialización de las asignaturas y la posible presencia de estudiantes de otros países, los elementos TIC se diseñan tanto en español como en inglés utilizando la misma herramienta. 
Todo el proceso ha sido diseñado para una participación anónima a través de la codificación del alumnado. El código es asignado a cada persona de forma aleatoria y confidencial, así permite el rastreo de la evolución del comportamiento sin comprometer los datos personales de los estudiantes.

A continuación se describen los distintos elementos que componen el proyecto.

\subsection{Guía}

Esta guía tiene por objeto orientar al docente en la aplicación de las herramientas desarrolladas en el marco del proyecto. Su contenido se detalla a continuación:

\section{Sesión 1}

1. Se introduce el objetivo de la sesión y se solicita la colaboración del alumnado.

2. Con el fin de medir la evolución de la adquisición de los conceptos relacionados con sostenibilidad a trabajar en la asignatura, se pide a los estudiantes que completen una encuesta de forma individual y anónima, a traves de Google Forms ${ }^{\circledR}$. Para ello, cada alumno tendrá asignado un identificador numérico único que asegurará el anonimato de sus respuestas y que deberán utilizar en las distintas fases. El tiempo estimado para rellenar el cuestionario es de 15 minutos y se proporcionarán dos versions del mismo: en castellano y en inglés.

3. El profesorado plantea la pregunta “¿Cuál creéis que es el tema principal de la encuesta?”. De esta manera, se pretende que el alumnado identifique los diferentes aspectos de la sostenibilidad trabajados y la importancia de los mismos.

4. Mediante la herramienta de gamificación Kahoot, se realiza un juego en el que se trabajan los distintos pilares de la sostenibilidad. Se estima una duración de unos 10 minutos para responder a 25 preguntas. Posteriormente, se repasarán los resultados de todas las preguntas. El tiempo total estimado es de 1 hora.

5. Se plantea un pequeño debate (5 minutos de duración aproximadamente), que se iniciará con la pregunta: ¿cómo podemos contribuir al Desarrollo Sostenible? Se abordará desde dos puntos de vista:

○ A título profesional (con formación, con orientación de nuestro trabajo, en la toma de decisiones, cuando tengamos capacidad para ello, etc.)

○ A título personal (reciclaje, movilidad, etc.)

6. A continuación, se plantea una pequeña encuesta de movilidad, utlizando una aplicación web, donde los alumnos deberán reportar la movilidad del día anterior (todas las actividades y los desplazamientos realizados).

7. Una vez completada la encuesta, trabajando en parejas, se realiza un pequeña actividad. Para ello, se pide a los estudiantes que seleccionen uno de los viajes realizados el día anterior que no sea sostenible según su punto de vista y que propongan una alternativa más sostenible y que sea viable. Seguidamente, deberán completar una ficha, evaluando ambas alternativas de transporte 
en función de su contribución a la movilidad sostenible, asignando un valor (del 1 al 5) a cada uno de los siguientes factores:
○ Contaminación
- Consumo energético
○ Ruido
- Ocupación del espacio
○ Precio
○ Tiempo
- Bienestar físico
- Nivel de estrés
○ Afección a otros usuarios

\section{Sesión 2}

8. Repetición de la encuesta realizada al inicio de la asignatura, con el fin de evaluar el grado de adquisición de los conocimientos relacionados con sostenibilidad, así como otros aspectos relacionados con el grado de sensibilización a distintos niveles: personal y profesional.

A continuación, se describen con mayor detalle los instrumentos y herramientas mencionados anteriormente en el que se emplea el uso de TICs.

\subsection{Encuesta (inicial y final)}

El objetivo de la encuesta es evaluar el grado de conocimiento inicial de los estudiantes de la sostenibilidad, así como el grado de sensibilización a distintos niveles: personal y profesional. El cuestionario se lleva a cabo a través de Google Forms ${ }^{\circledR}$, por ser un instrumento sencillo para la realización de encuestas, de uso gratuito y adaptado para uso en plataformas móviles (tabletas y teléfonos inteligentes).

La encuesta se compone de las siguientes secciones:

- Introducción. En esta sección se introduce brevemente el objetivo de la encuesta y el proyecto en el que se enmarca. Así mismo, se incluyen los datos de contacto de las personas involucradas en el estudio.

- Datos sociodemográficos (sexo, edad, ingresos, lugar de residencia habitual y durante el curso, ingresos, etc.). Para ello, se emplearon 7 ítems.

- Datos básicos sobre movilidad: disponibilidad de vehículo privado, bicicleta y abonos de transporte público. En este caso se utilizan 3 ítems, la disponibilidad se mide utilizando la escala: nula (nunca), baja ( 1 o 2 días a la semana), media (3 o 4 días a la semana), alta (5 días a la semana o más). Por último, se pregunta por los abonos de transporte público de los que se dispone, para lo cual se proporciona un listado cerrado, con las categorías: alquiler de bicicleta pública, sistema de moto compartida, abono de transporte público (bus, metro, combinado...) u otros.

- Importancia de la sostenibilidad en la vida diaria. Se utilizan 4 ítems, en los que se pregunta por la frecuencia de reciclaje, uso de bolsas personales para la compra, participación en voluntariado y planificación anual de los ahorros. Se proporciona respuesta abierta, en escala temporal (nunca, casi nunca, a veces, casi siempre y siempre) 
- Conocimiento sobre el concepto de Desarrollo Sostenible. Se emplea una pregunta de respuesta múltiple, en la que se deben seleccionar todos los factores que forman para del Desarrollo Sostenible para la persona encuestada. Dichos factores son: recursos naturales, salud, innovación, finanzas, bienestar social, economía, accesibildad, vivienda y gobierno.

\subsection{Juego en Kahoot ${ }^{\circledR}$}

Kahoot! es una conocida herramienta de gamificación basada en una aplicación web que permite la realización de juegos, recompensando a quienes progresan en las respuestas adecuadamente con una mayor puntuación. Esta herramienta se ha popularizado durante los últimos años y su uso en el aula se ha extendido ampliamente en el entorno universitario. La gran mayoría de estudiantes está familiarizado con ella.

Para realizar la actividad, el profesorado proyecta las preguntas, mientras que los estudiantes contestan a desde sus dispositivos (teléfono móvil, tableta, ordenador, etc.). Es necesario contar con una conexión a internet estable.

El juego planteado consta de 24 preguntas sobre el grado de conocimiento del concepto de Sostenibilidad, diferencias entre recursos renovables y no renovables, huella ecológica, huella de carbono, energía y sostenibilidad alimentaria.

En este caso, se opta por un juego tipo "quiz" cronometrado, en la Figura 1 se muestra el aspecto visual del juego a través de la captura de pantalla de dos de las preguntas.
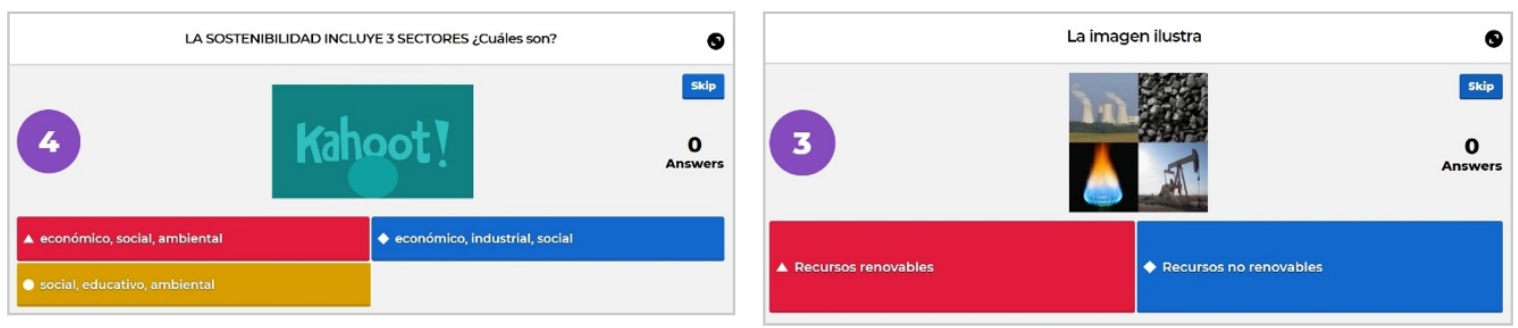

Fig. 1 Capturas de pantalla del juego realizado con Kahoot.

Manteniendo la duplicidad por idiomas, el juego se diseña tanto en español como en inglés; y se proyecta simultáneamente en clase utilizando el modo de pantalla partida.

\subsection{Encuesta de movilidad a través de aplicación web}

En esta actividad se pretende que los estudiantes reflexionen sobre su movilidad realizando una pequeña encuesta. Posteriormente, se realiza de forma conjunta una debate sobre la sostenibilidad de sus desplazamientos.

Se parte una aplicación web desarrollada a medida en la UPV en el marco del proyecto MINERVA (TRA2015-71184-C2-1-R), financiado por el Ministerio de Economía, Industria y Competitividad y que fue adaptada para el presente estudio. La aplicación consiste en una interfaz web, en la que se pide a los estudiantes que reporten todos los desplazamientos y actividades realizados durante el día anterior, así como las principales características de los mismos (hora de inicio y fin, lugar de realización de la actividad u origen/destino del viaje, modo de transporte o motivo del desplazamiento). La aplicación muestra un esquema del diario de día, tal como se puede ver en la Figura 2, que mostrarán a la persona con la que trabajan en parejas para inciciar el debate y realizar la actividad sobre la evaluación de la movilidad teniendo en cuenta criterios de sostenibilidad. 


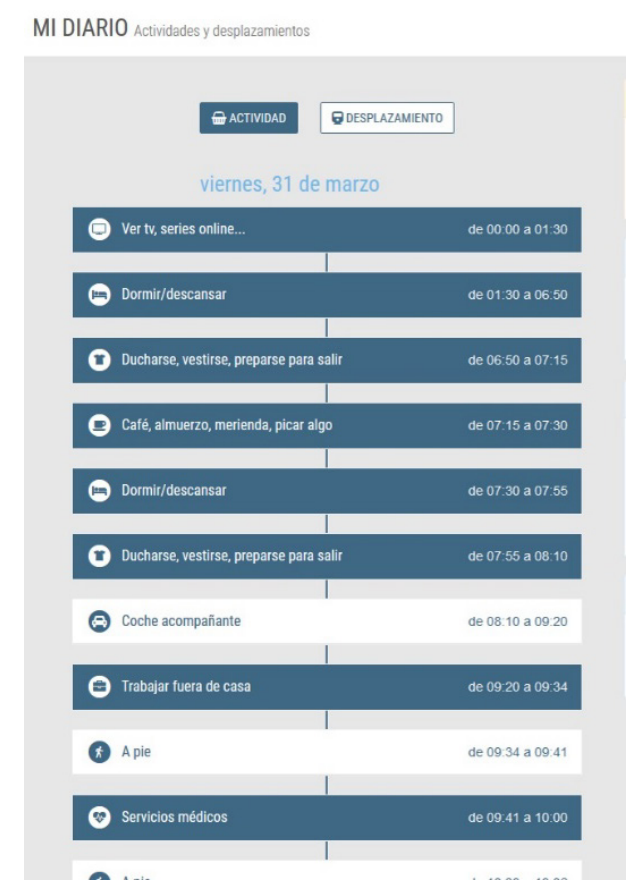

Fig. 2 Capturas de pantalla de la aplicación web utilizada para caracterizar la movilidad

\section{Conclusiones}

La metodología propuesta está todavía en fase de aplicación, no obstante los resultados que se evidencian por el momento son de carácter positivo.

El uso de herramientas de acceso gratuito y adaptadas para aplicaciones móviles, ha permitido una elevada participación superando el $90 \%$ de alumnos matriculados en el total de la asignatura. Sin embargo, la necesidad de trabajar en dos idiomas en paralelo en alguna de las asingaturas, ha supuesto una dificultad añadida a la hora de llevar a cabo el juego en Kahoot ${ }^{\circledR}$ por la necesidad de sincronizar dos partidas en una misma pantalla.

En relación a la encuesta de movilidad, el anonimato en el proceso ha resultado un aspecto fundamental. Los alumnos han manifestado su conformidad a compartir datos de carácter personal como son los movimientos que realizan fuera del ámbito educativo.

Este proyecto ha permitido desarrollar una metodología sólida y aplicable a sucesivos cursos académicos facilitando la incorporación de conocimientos transversales vinculados al Desarrollo Sostenible a través de la gamificación y las herramientas TICs. Así, las competencias transversales tales como el análisis y resolución de problemas (CT3, UPV), la responsabilidad ética, medioambiental y profesional (CT7, UPV), el pensamiento crítico (CT9, UPV), el conocimiento de problemas contemporáneos (CT10, UPV), y las competencias informáticas e informacionales (UA) quedan integradas en la formación en torno al concepto universal de Desarrollo Sostenible independiente de la titulación en la que se imparta.

Los trabajos futuros a desarrollar a raíz de este proyecto incluyen la evaluación de los resultados tras aplicar la metodología propuesta, así como el análisis de la viabilidad de aplicación de la metodología a asignaturas de otros ámbitos. 


\section{Agradecimientos}

Este trabajo se realizó en el marco de la red "La inclusión del uso de las TICs como medio de instrucción en el desarrollo de habilidades y competencias en el ámbito de los estudios de la sostenibilidad en el aula" del Instituto de Ciencias de la Educación de la Universidad de Alicante (curso 2019/2020), código 4947.

\section{Referencias}

Álvarez Romero, C. \& Osete Cortina, L. (2019). Game-based learning: juego de cartas para aprender Ciencia. Congreso In-Red 2019 UPV, 11 y 12 de julio de 2019 Doi:http://dx.doi.org/10.4995/INRED2019.2019.10404

Annan-Diab, F. \& Molinari, C. (2017). Interdisciplinarity: Practical approach to advancing education for sustainability and for the Sustainable Development Goals. The International Journal of Management Education, 15, 73-83. Recuperado de: https://www.sciencedirect.com/science/article/abs/pii/S1472811717300939.

Chaiyo, Y. \& Nokham, R. (2017). "The Effect of Kahoot, Quizizz and Google forms on the Student's Perception in the Classrooms Response System”. International Conference on Digital Arts, Media and Technology (ICDAMT) Digital Economy for Sustainable Growth. pp. 178-182. Chiang Mai.

García-García, D., Carbonell-Verdú, A., Montañés, N., Quiles, L. \& Fombuena, V. (2017). Incorporación de la aplicación Kahoot! para la evaluación de las prácticas de la asignatura de "Ciencia de Materiales". III Congreso Nacional de innovación educativa y de docencia en red. Editorial Universitat Politècnica de València, 1209-1217. Doi:10.4995/INRED2017.2017.6799

GOOGLEFORMS < https://www.google.com/forms/about/> [Consulta: 23 de marzo de 2020]

Guardia, J.J., Del Olmo, J.L., Roa, I. \& Berlanga, V. (2019). "Innovation in the teaching learning process: the case of Kahoot!", en On the Horizon, vol. 27, issue 1, p. 35-45.

Hernández-Crespo, C., AV Pachésa, M., Romero, I., Mengual, J., González, J.A., Barata, R., Paredes, J., Serralta \& J. (2019). Diseño, aplicación y evaluación de metodologías activas de aprendizaje, utilizando TIC en el ámbito de la ingeniería ambiental. Congreso In-Red 2019 UPV, 11 y 12 de julio de 2019. Doi:http://dx.doi.org/10.4995/INRED2019.2019.10465

Jardim, E., Serrano Torregrosa, E., Linares, N. \& Silvestre-Albero, A. (2016). Nuevas herramientas didácticas para acerca la Química Sostenible a la vida cotidiana del alumnado. Llibre d'actes de les XV Jornades de Xarxes d'Investigació en Docència Universitària-XARXES 2017 i I Workshop Internacional d'Innovació en Ensenyament Superior i TIC-INNOVAESTIC 2017 / Libro de actas de las XV Jornadas de Redes de Investigación en Docencia Universitaria- REDES 2017 y I Workshop Internacional de Innovación en Enseñanza Superior y TICINNOVAESTIC 2017, 1, 366-367. Recuperado de: (https://web.ua.es/es/ice/redes-innovaestic2017).

KAHOOT!. <https://kahoot.com/> [Consulta: 23 de marzo de 2020]

Ojeda-Barceló, F., Gutiérrez-Pérez, J. \& Perales-Palacios, F. J. (2011). TIC y Sostenibilidad: Obstáculos y posibilidades para los educadores ambientales. Profesorado. Revista de Currículum y Formación de Profesorado, 15(1), 263-313.

Pastor Villa, R. (2019). La implementación de la herramienta Kahoot en el aula universitaria. Congreso In-Red 2019 UPV, 11 y 12 de julio de 2019. Doi:http://dx.doi.org/10.4995/INRED2019.2019.10411 2019, Universitat Politècnica de València.

Reyes-Sánchez, L.B. (2012). Aporte de la química verde a la construcción de una ciencia socialmente responsable. Educación Química, 23(2), 222-229.

Ruiz, M.C, Martínez, R., García, E., Pedrosa, C. \& Licerán, A. (2019). ¿Es divertido aprender con Kahoot!?: la percepción de los estudiantes. Congreso In-Red 2019 UPV, 11 y 12 de julio de 2019. Doi: http://dx.doi.org/10.4995/INRED2019.2019.10368 2019, Universitat Politècnica de València. 
Sainz-de-Abajoa, B., De la Torre-Díeza, I., López-Coronado, M., Aguiar Péreza, J.M \& De Castro Lozano, C. (2019). Aplicación plural de herramientas para gamificar. Análisis y comparativa. Congreso In-Red 2019 UPV, 11 y 12 de julio de 2019 Doi:http://dx.doi.org/10.4995/INRED2019.2019.10467 2019, Universitat Politècnica de València 